Published in final edited form as:

J Matern Fetal Neonatal Med. 2018 November ; 31(22): 3014-3020. doi:

$10.1080 / 14767058.2017 .1362384$.

\title{
Short interpregnancy interval increases the risk of preterm premature rupture of membranes and early delivery
}

\author{
Raj Shree ${ }^{\mathrm{a}}$, Aaron B. Caughey ${ }^{\mathrm{b}}$, and Suchitra Chandrasekaran ${ }^{\mathrm{a}}$ \\ aDepartment of Obstetrics and Gynecology, Division of Maternal Fetal Medicine, University of \\ Washington Medical Center, Seattle, WA, USA \\ bDepartment of Obstetrics and Gynecology, OHSU, Portland, OR, USA
}

\begin{abstract}
Objectives-Preterm premature rupture of membranes (PPROM) is a major contributor to overall preterm birth (PTB) rates. A short interpregnancy interval (IPI) is a well-known risk factor for PTB. It is unknown if a short IPI specifically affects the risk of developing PPROM in a subsequent pregnancy. We sought to determine the association between IPI and the risk of PPROM in a subsequent pregnancy.

Methods-A retrospective cohort study using the Missouri birth certificate database of singleton births from 2003 to 2013 was conducted. A short IPI (delivery of the prior pregnancy to conception of the index pregnancy) was defined as $\leq 6$ months. IPI $>6$ months was categorized into two groups: IPI 7-23 months and IPI $\geq 24$ months. PPROM was defined as premature rupture of membranes between $16^{0}$ and $36^{6}$ weeks. Multivariable logistic regression was conducted to determine the association between IPI and PPROM while controlling for maternal age, race, body mass index (BMI), education level, use of social services (Medicaid insurance, food stamps, or participation in the WIC [Women, Infants, and Children] program), tobacco use, and history of PTB. Secondary outcome included the gestational age at delivery, categorized into five subgroups ( $ک 24^{0}, 24^{1}-28^{0}, 28^{1}-32^{0}, 32^{1}-34^{0}$, and $34^{1}-36^{6}$ weeks).
\end{abstract}

Results-474,957 subjects with singleton gestations had data available to calculate the IPI. Of these, $1.4 \%$ ( $n=6797$ ) experienced PPROM. IPI $₫ 6$ months was significantly associated with an increased risk of developing PPROM compared with patients with IPI $\geq 24$ months (odds ratio (OR) $1.80,95 \%$ CI 1.70-1.90, $p<.001$ ). A higher proportion of women with IPI $\$ 6$ months delivered between $28^{1}$ and $32^{0}$ weeks compared to the other two IPI groups (27.0 versus 15.0 and $16.4 \%, p<.001)$. Individual maternal factors associated with an increased risk of PPROM included advanced maternal age, African American race, BMI $<18.5 \mathrm{~kg} / \mathrm{m}^{2}, \mathrm{BMI} \geq 30 \mathrm{~kg} / \mathrm{m}^{2}$, use of social services, tobacco use, and a prior PTB.

Conclusion-Our data demonstrate that an IPI of $\$ 6$ months is significantly associated with an increased risk of developing PPROM in the subsequent pregnancy. Of greater clinical relevance is

CONTACT Raj Shree, shreer23@uw.edu, Department of Obstetrics and Gynecology, University of Washington Medical Center, 1959 NE Pacific Street, Seattle, WA 98195, USA.

Disclosure statement

The authors report no conflict of interest. The authors have no financial disclosures to report. 
that these women were more likely to deliver between $28^{1}$ and $32^{0}$ weeks as compared with women with a longer IPI. Novel to this study is the establishment of a specific link between a short IPI and PPROM with subsequent early delivery. Several maternal demographic factors known to be associated with PTB risk were also found to be associated with an increased risk of PPROM. Further studies are necessary to elucidate plausible biologic mechanisms ultimately leading to the development and implementation of preventive and therapeutic strategies for this high-risk cohort.

\section{Keywords}

Interpregnancy interval; preterm birth; preterm premature rupture of membranes

\section{Introduction}

Preterm premature rupture of membranes (PPROM) contributes significantly to overall preterm birth (PTB) rates as well as increasing neonatal morbidity and mortality. Recent vital statistics data for the USA (2014) report a PTB rate (<37 weeks) of 9.57\% [1,2], with PPROM underlying 25-30\% of the cases [3].

It is also well established that a short interpregnancy interval (IPI), often defined as $\$ 6$ months, is associated with poor obstetric and neonatal outcomes including PTB, low birth weight, small for gestational age, fetal death, and early neonatal death [4-6]. There are currently no established recommendations for optimal birth spacing in the USA. The WHO recommends an interval of 24 months following a live birth prior to conception of the next pregnancy, citing a reduction in the risk of adverse maternal, perinatal, and infant outcomes with this strategy [7].

A study from the Utah birth certificate data was one of the first sources to establish the independent effect of IPI on adverse birth outcomes by controlling for important medical and sociodemographic confounders. The authors found that an interval of 18-23 months was associated with the lowest risk of their primary outcomes: low birth weight $(<2500 \mathrm{~g})$, PTB ( $<37$ weeks), and small for gestational age (birth weight $<10$ th percentile for gestational age). Furthermore, they found a significant increase in these adverse outcomes with shorter interpregnancy intervals of $0-5$ months (low birth weight odds ratio (OR) 1.4, 95\% CI 1.31.6; PTB OR 1.4, 95\% CI 1.3-1.5; small for gestational age OR 1.3, 95\% CI 1.2-1.4) [4].

More recent studies have also highlighted the importance of IPI on the risk of adverse perinatal outcomes in patients with both prior term and preterm births [5,6]. A large cohort study of women with a prior term birth $(n=69,055)$ found that a short IPI ( $<6$ months) was associated with an increased risk of extreme PTB (24-32 weeks) [OR 2.2, 95\% CI 1.4-3.6], moderate PTB (33-36 weeks) [OR 1.6, 95\% CI 1.3-2.0], and neonatal death (OR 3.6, 95\% CI 1.2-10.7) in the subsequent pregnancy) [5]. In patients with a prior PTB, there were similar findings with an increased risk of recurrent PTB in subsequent pregnancies following an IPI of $<6$ months (OR 1.44, 95\% CI 1.2-1.8) [6].

Although the role of IPI is well established with respect to the risk of PTB as a whole, it is unclear whether a short IPI specifically increases the risk of developing PPROM in a subsequent pregnancy. The available data to date have not addressed this particular subset of 
patients. Hence, we sought to determine the impact of different clinically relevant IPI intervals on the risk of PPROM in subsequent pregnancies. We also sought to investigate the gestational age at delivery of these patients.

\section{Materials and methods}

We conducted a retrospective cohort study of singleton deliveries between 2003 and 2013, utilizing the Missouri Birth Certificate deidentified database, which included all births and fetal deaths in the state of Missouri. We included only singleton gestations with delivery date available for the current and prior pregnancy. The Institutional Review Board of the University of Washington deemed the study exempt.

IPI, defined as the time from delivery of the last pregnancy to conception of the current pregnancy, was calculated using the following data points: gestational age at delivery of current pregnancy, prior pregnancy delivery year, and current pregnancy delivery year. Because we received a completely deidentified database, exact birthdates were not available and thus we used the calculation presented below.

(Year of current birth - Year of last birth) $\times$ $52=$ Interdelivery interval (in weeks)

$I P I=$ Interdelivery interval - gestational age at delivery of current birth

For patients with the same year listed for their last and current delivery, we calculated the IPI by subtracting the gestational age at delivery from 52 weeks. This method assigned the longest IPI possible to these cases. We then identified women whose current pregnancy was complicated by PPROM, defined as rupture of membranes $>12$ hours with delivery between

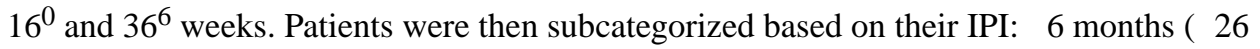
weeks), 7-23 months (27-103 weeks), and $\geq 24$ months ( $\geq 104$ weeks).

We also looked at individual maternal sociodemographic factors across each IPI group to determine the individual risk for PPROM conferred by each factor. Maternal race was characterized as Caucasian, African American, Hispanic, or Asian if only this race was designated for that individual. If more than one race was designated, or if a woman identified herself as American Indian, Pacific Islander, or other, she was placed in our "Other" category. We then investigated the gestational age at delivery among the three IPI groups in a variety of clinically relevant gestational age categories: $24^{0}$ weeks, $24^{1}-28^{0}$ weeks, $28^{1}-32^{0}$ weeks, $32^{1}-34^{0}$ weeks, and $34^{1}-36^{6}$ weeks.

We hoped to investigate neonatal outcomes such as Apgar score, neonatal intensive care unit (NICU) admission, respiratory distress syndrome (RDS), assisted ventilation, and fetal/ neonatal death. Unfortunately, exploration of these variables revealed these to be unreliably coded. For example, information regarding NICU admission and assisted ventilation were only coded in $35 \%$ of subjects $(n=165,628)$. Similarly, the presence of absence of RDS was 
only coded in 65\% of subjects. Although Apgar score and fetal/neonatal death were available for all subjects, we did not include this in our analysis given the overall limited picture of neonatal outcomes this represents and that no meaningful conclusions could be drawn.

Categorical variables were compared using $\chi^{2}$ and Fisher's exact tests as appropriate. Continuous variables were tested for normality using the Shapiro-Wilk test and were compared using the $t$-test and Kruskal-Wallis tests as appropriate. Multivariable logistic regression was performed to determine the association between IPI and PPROM.

Confounders in the model included maternal age, race, body mass index (BMI), use of social services (use of Medicaid insurance, food stamps, or participation in the Women, Infants, and Children [WIC] program), tobacco use, and a history of PTB. Variables were included in our model either because they are commonly accepted as important confounders (maternal age, race, surrogates of socioeconomic status) or because they are previously established risk factors for adverse pregnancy outcomes (BMI, tobacco use). Because a history of PTB is a critically important variable for our primary outcome, we forced this variable into our model.

\section{Results}

474,957 patients with singleton gestations had data available to calculate the IPI. Of these, $1.4 \%(n=6797)$ experienced PPROM. The overall preterm birth rate for the population of women who did not experience PPROM was $8.7 \%(n=40,686)$. The incidence of PPROM was $2.2 \%(n=2104), 1.2 \%(n=1566)$, and $1.2 \%(n=3127)$ for IPI 56 months, IPI $7-23$ months, and IPI $\geq 24$ months, respectively. The incidence of PPROM was significantly higher in patients with IPI $\$ 6$ months compared with those with IPI 7-23 months and IPI $\geq 24$ months $(p<.001)$.

Among pregnancies complicated by PPROM, women with an IPI $\geq 24$ months tended to be older and had a higher rate of obesity (BMI $230.0 \mathrm{~kg} / \mathrm{m}^{2}$ ) (Table 1). A higher rate of use of social services was seen in the IPI 7-23 months group, whereas tobacco use and a history of prior PTB were more common among women with IPI $\geq 24$ months (Table 1).

\section{Association between IPI and PPROM}

Multivariable logistic regression demonstrated a significantly increased risk of developing PPROM in a subsequent pregnancy among those with IPI $\$ 6$ months (OR 1.84, 95\% CI $1.74-1.95, p<.001)$ compared with women with IPI $\geq 24$ months. There was no statistically significant increase in the risk of developing PPROM following IPI 7-23 months (OR 1.05, 95\% CI 0.99-1.12, $p=.10$ ) (Table 2).

\section{Evaluation of demographic characteristics for women with PPROM by IPI}

In the evaluation of individual maternal sociodemographic and obstetric factors with respect to the risk of PPROM, we found that maternal age 35-44 years, African American race, BMI $<18.5 \mathrm{~kg} / \mathrm{m}^{2}$, BMI $\geq 30 \mathrm{~kg} / \mathrm{m}^{2}$, use of social services, tobacco use, and a history of PTB were all independently associated with an increased risk of developing PPROM in a subsequent pregnancy (Table 3). 


\section{Association between IPI and gestational age at delivery among those with PPROM}

A significantly higher percentage of patients with IPI $\$ 6$ months delivered between $28^{1}$ and $32^{0}$ weeks $(27.1 \%$ versus $14.7 \%$ and $16.6 \%, p<.001)$ and $32^{1}-34^{0}$ weeks $(30.0 \%$ versus $22.7 \%$ and $27.8 \%, p<.001$ ) compared with the other IPI groups. A higher percentage of patients with IPI 7-23 months delivered at 2 - 4 weeks (20.0\%) and $24^{1}-28^{0}$ weeks (12.1\%) compared with women with IPI $\$ 6$ months $(0.0 \%$ and $10.3 \%$, respectively) and IPI $\geq 24$ months (6.6\% and 9.5\%, respectively), $p<.001$ and $p=.02$. A significantly higher percentage of patients with IPI $\geq 24$ months delivered between $34^{1}$ and $36^{6}$ weeks (39.4\%) compared with the other IPI groups (32.6\% and 30.5\%, $p<.001)$ (Table 4).

\section{Discussion}

It is well known that a short interval between pregnancies increases the risk of PTB in a subsequent pregnancy. Indeed, one of the Healthy People 2020 objectives put forth by the Department of Health and Human Services calls for a 10\% reduction in the number of pregnancies conceived within 18 months of a previous birth [8]. Novel to our study is the establishment of a specific link between shorter IPI and an increased risk of PPROM in a subsequent pregnancy. We found a rate of $1.4 \%$ for PPROM in our population, which is roughly half the rate quoted in the literature [3]. We suspect that coding errors contribute to this decreased rate, especially in women who may have had ROM $>12 \mathrm{~h}$, but went on to deliver in the next $24-48 \mathrm{~h}$, or in the extremely preterm gestational ages (16-24 weeks).

Of particular importance is the finding of an increased incidence of deliveries between $28^{1}$ and $32^{0}$ weeks following a short IPI ( $\$$ months). This suggests that not only are these women at an increased risk of PPROM in their subsequent pregnancies, but a higher proportion of these women deliver in the very preterm gestational age range, compared with women with a longer IPI. Interestingly, we also found a significantly higher risk of delivery at $\mathcal{2} 4$ weeks in women with an IPI of 7-23 months. Because the lowest risk of adverse birth outcomes likely occurs following an IPI of 18-23 months [4,9], it is possible that our IPI grouping of 7-23 months encompasses a mixed group with anticipated diverse outcomes. Women with an IPI of 7-18 months likely represent a different risk group than women with an IPI of 18-23 months. As such, interpretation of the outcomes for the IPI 7-23 months group is likely mathematically correct, but may be misleading given possible opposing biological behavior.

Regardless, an increase in deliveries in this gestational age range is acutely relevant. As our definitions for periviability and viability are evolving, delivery in this time frame involves complex decision making for patients and providers $[10,11]$. The inherent morbidity and mortality to the periviable neonate are apparent $[12,13]$; however, the implications to maternal reproductive health, particularly related to classical cesarean delivery (CD), are important considerations and require thoughtful deliberation. Even if a classical CD can be avoided, a recent study has shown that periviable low transverse CDs are associated with an increased risk of uterine rupture in subsequent pregnancies compared to low transverse $\mathrm{CD}$ performed at term (1.8\% versus $0.4 \%$; OR $4.7,95 \%$ CI 1.7-13.4, $p=.004)$ [14]. 
The pathophysiology behind the risk of PPROM in a subsequent pregnancy following a shorter IPI remains unclear. Serial cervical biopsies after a term delivery over a 15-month period revealed that cervical collagen concentrations increase gradually after a term birth with significant changes noted up to 9 months, but insignificant increases in the 9-15-month period [15]. This suggests that a short IPI (especially $\$ 6$ months) may contribute to incomplete cervical remodeling as a mechanism for cervical shortening in a subsequent pregnancy, thus potentially increasing the risk for preterm membrane rupture.

With respect to individual factors, we found that women of advanced maternal age (AMA) had an increased risk of PPROM compared to younger women. The link between AMA and PTB has previously be suggested [16], and here we show an association between AMA and the risk of PPROM. This risk must be weighed against the decline in fertility with advancing age for women desiring future pregnancies, and thus should be included in individualized counseling.

African American race was strongly associated with developing PPROM. Racial disparities in obstetric outcomes are well documented in the USA and non-Hispanic African American women are disproportionately affected by these with respect to PTB and neonatal outcomes [3]. IPI is thus a modifiable risk factor to reduce both PTB and PPROM in subsequent pregnancies in this at risk population.

Extremes of BMI are associated with PTB risk [17] and some studies suggest a link between obese gravidas $\left(\mathrm{BMI}>30 \mathrm{~kg} / \mathrm{m}^{2}\right.$ ) and delivery at $<37$ weeks and $<34$ weeks with PPROM [18], as seen in our analysis. It is not surprising that our data support an association between tobacco use and PPROM as this is a known risk factor [19] and is additionally linked with PTB risk [20,21]. Lower socioeconomic status, judged by the use of social services in our analysis, is also a well-established risk factor for PTB [22,23], and thus it is biologically plausible for this to also be associated with the risk of PPROM.

Strengths of our study include data collection from a large cohort of women over a 10-year period with control of many important covariates. Importantly, we are able to control for a known significant contributor to PTB, a history of a prior preterm delivery, to determine the potential impact of IPI itself on the risk of PPROM and gestational age at delivery in our case patients.

Our study is not without limitations. By nature, it is dependent on the quality of data captured in birth certificate entries and its retrospective nature does not allow control for certain potential confounders. Without the actual birth date, misclassification of the IPI is possible, however, we took measures to avoid underestimation when possible. Although an intrinsic limitation of this database, given the large number of subjects $(>400,000)$ and that all pregnancies are from within a defined period, we may reasonably expect the rate of misclassification to be uniform across the board. It is possible that mothers are included multiple times for successive births during this 10-year period; as such, we cannot confidently assume that all observations are independent. Additional prospective obstetrical databases which include our parameters of interest are available on a national level in the 
USA, Canada, and Australia, and should be considered for use for further studies, especially to parse out naunces we were unable to uncover.

We found no deliveries at $\_24$ weeks in our PPROM patients with IPI $\$ 6$ months. Conversely, we found a large proportion of patients (20\%) with deliveries in this gestational age group for IPI 7-23 months. Several methods of reanalysis of the data and by multiple authors (RS and SC) confirmed this finding. We suspect that these cases of PPROM did not get coded as such, especially in the very preterm gestational age groups (16-22 weeks). This may be true given that the database was collected from 2003 to 2013, during which time viability was more firmly accepted to be 24-weeks gestation, and thus pregnancies with ROM prior to this may not have been categorized as PPROM cases by individuals completing the birth certificate. A validation study of New York City and Vermont 2009 birth certificate data found that sensitivity and positive predictive value for correct documentation of PPROM was poor ( $<70 \%$ for both) [24]. This analysis falls within the time period of the database we used and may explain our unusual results in the $\_24$ weeks delivery group.

The exact indication for delivery in each case is also not known. As such, it is challenging to determine the mechanism by which a short IPI contributes to early deliveries in pregnancies complicated by PPROM. We are also unaware of the gestational age at which PPROM occurred, and thus cannot comment on the length of latency in these pregnancies. However, patients with PPROM delivering between $34^{1}$ and $36^{6}$ weeks likely experienced PPROM in this gestational age window as it is standard practice in the USA to proceed with delivery if PPROM occurs after 34 weeks [25]. Thus, it can be suggested from our data that if PPROM occurred in patients with an IPI $\geq 24$ months, it was significantly more likely to occur after 34 weeks, compared with women with a shorter IPI (Table 4).

We explored the available neonatal data, however, due to many missing data points, deemed this analysis too weak for inclusion as no meaningful conclusions could be drawn. We anticipate gestational age at delivery to be the primary driver for adverse neonatal outcomes, and thus anticipate poorer consequences for neonates born to women with IPI $₫ 6$ months compared with those with a longer IPI; however, further research is necessary to confirm this supposition.

Our data clearly demonstrates that women are at increased risk of PPROM following a short IPI ( $\$ 6$ months), and that this subset of patients is at particularly high risk for earlier preterm deliveries and thus represents an at-risk group who warrant targeted counseling and therapies. Given the risk of delivery in the periviable and extreme preterm gestational age ranges, and the relative affordability of highly effective long acting reversible contraception, the public health significance and cost-burden of these cases are significant and warrant further research.

\section{Acknowledgments}

Poster presentation at the 36th Annual Pregnancy Meeting, Society for Maternal Fetal Medicine, Atlanta, GA, 16th February 2016. 


\section{References}

1. Hamilton BE, Martin JA, Osterman MJ, et al. Births: final data for 2014. Natl Vital Stat Rep. 2015; 64(12):1-64.

2. Ananth CV, Joseph KS, Oyelese Y, et al. Trends in preterm birth and perinatal mortality among singletons: United States, 1989 through 2000. Obstet Gynecol. 2005; 105(5 Pt 1):1084-1091. [PubMed: 15863548]

3. Goldenberg RL, Culhane JF, Iams JD, et al. Epidemiology and causes of preterm birth. Lancet. 2008; 371(9606):75-84. [PubMed: 18177778]

4. Zhu BP, Rolfs RT, Nangle BE, et al. Effect of the interval between pregnancies on perinatal outcomes. N Engl J Med. 1999; 340(8):589-594. [PubMed: 10029642]

5. Smith GC, Pell JP, Dobbie R. Interpregnancy interval and risk of preterm birth and neonatal death: retrospective cohort study. BMJ. 2003; 327(7410):313. [PubMed: 12907483]

6. DeFranco EA, Stamilio DM, Boslaugh SE, et al. A short interpregnancy interval is a risk factor for preterm birth and its recurrence. Am J Obstet Gynecol. 2007; 197(3):264.e1-264.e6. [PubMed: 17826413]

7. Report of a WHO technical consultation on birth spacingGeneva, Switzerland: Jun 13-15, 2005

8. Topics and objectives: family planningDepartment of Health and Human Services; 2010Healthy People 2020. Available from: healthypeople.gov

9. Conde-Agudelo A, Rosas-Bermúdez A, Kafury-Goeta AC. Birth spacing and risk of adverse perinatal outcomes: a meta-analysis. JAMA. 2006; 295(15):1809-1823. [PubMed: 16622143]

10. Raju TN, Mercer BM, Burchfield DJ, et al. Periviable birth: executive summary of a joint workshop by the Eunice Kennedy Shriver National Institute of Child Health and Human Development, Society for Maternal-Fetal Medicine, American Academy of Pediatrics, and American College of Obstetricians and Gynecologists. Obstet Gynecol. 2014; 123(5):1083-1096. [PubMed: 24785861]

11. Ecker JL, Kaimal A, Mercer BM, et al. Periviable birth. Obst Gynecol Surv. 2016; 71(3):137-139.

12. Backes $\mathrm{CH}$, Rivera BK, Haque U, et al. A proactive approach to neonates born at 23 weeks of gestation. Obstet Gynecol. 2015; 126(5):939-946. [PubMed: 26444114]

13. Rysavy MA, Li L, Bell EF, et al. Between-hospital variation in treatment and outcomes in extremely preterm infants. N Engl J Med. 2015; 372(19):1801-1811. [PubMed: 25946279]

14. Lannon SM, Guthrie KA, Vanderhoeven JP, et al. Uterine rupture risk after periviable cesarean delivery. Obstet Gynecol. 2015; 125(5):1095-1100. [PubMed: 25932837]

15. Sundtoft I, Sommer S, Uldbjerg N. Cervical collagen concentration within 15 months after delivery. Am J Obstet Gynecol. 2011; 205(1):59.e1-59.e3. [PubMed: 22088899]

16. Waldenström U, Cnattingius S, Vixner L, et al. Advanced maternal age increases the risk of very preterm birth, irrespective of parity: a population-based register study. BJOG. 2017; 124(8):12351244. [PubMed: 27770495]

17. Lynch AM, Hart JE, Agwu OC, et al. Association of extremes of prepregnancy BMI with the clinical presentations of preterm birth. Am J Obstet Gynecol. 2014; 210(5):428.e1-428.e9. [PubMed: 24321446]

18. Zhong Y, Cahill AG, Macones GA, et al. The association between prepregnancy maternal body mass index and preterm delivery. Am J Perinatol. 2010; 27(4):293-298. [PubMed: 19823961]

19. Castles A, Adams EK, Melvin CL, et al. Effects of smoking during pregnancy. Five meta-analyses. Am J Prev Med. 1999; 16(3):208-215. [PubMed: 10198660]

20. Andres RL, Day MC. Perinatal complications associated with maternal tobacco use. Semin Neonatol. 2000; 5(3):231-241. [PubMed: 10956448]

21. Cnattingius S. The epidemiology of smoking during pregnancy: smoking prevalence, maternal characteristics, and pregnancy outcomes. Nicotine Tob Res. 2004; 6(Suppl 2):S125-S140. [PubMed: 15203816]

22. Smith LK, Draper ES, Manktelow BN, et al. Socioeconomic inequalities in very preterm birth rates. Arch Dis Child Fetal Neonatal Ed. 2007; 92(1):F11-F14. [PubMed: 16595590] 
23. Thompson JM, Irgens LM, Rasmussen S, et al. Secular trends in socio-economic status and the implications for preterm birth. Paediatr Perinat Epidemiol. 2006; 20(3):182-187. [PubMed: 16629692]

24. Dietz P, Bombard J, Mulready-Ward C, et al. Validation of selected items on the 2003 U.S. standard certificate of live birth: New York City and Vermont. Public Health Rep USA: Standard. 2015; 130(1):60-70.

25. American College of Obstetricians and Gynecologists' Committee on Practice BulletinsObstetrics. Practice bulletin No. 172: premature rupture of membranes. Obstet Gynecol. 2016; 128(4):e165-e177. [PubMed: 27661655] 


\section{Table 1}

Maternal characteristics of patients with PPROM based on IPI $(N=6707)$.

\begin{tabular}{|c|c|c|c|c|}
\hline Characteristic & $\begin{array}{l}\text { IPI } \mathbf{6} \\
\text { months } \\
(N=\mathbf{2 1 0 4})\end{array}$ & $\begin{array}{l}\text { IPI 7-23 } \\
\text { months } \\
(N=1566)\end{array}$ & $\begin{array}{l}\text { IPI } \geq 24 \\
\text { months } \\
(N=3127)\end{array}$ & $p$ value \\
\hline \multicolumn{5}{|l|}{ Maternal age (years) } \\
\hline$\leq 35$ & $1862(88.5 \%)$ & $1385(88.4 \%)$ & $2482(79.4 \%)$ & $<.001$ \\
\hline $36-44$ & $240(11.4 \%)$ & $181(11.6 \%)$ & $636(20.3 \%)$ & \\
\hline$\geq 45$ & $2(0.1 \%)$ & $0(0 \%)$ & $9(0.3 \%)$ & \\
\hline \multicolumn{5}{|l|}{ Maternal race } \\
\hline Caucasian & $1403(66.7 \%)$ & $1015(64.8 \%)$ & $1969(63.0 \%)$ & .005 \\
\hline African American & $560(26.6 \%)$ & $423(27.0 \%)$ & $858(27.4 \%)$ & \\
\hline Hispanic & $2(0.1 \%)$ & $5(0.3 \%)$ & $5(0.2 \%)$ & \\
\hline Asian & $24(1.1 \%)$ & $11(0.7 \%)$ & $36(1.1 \%)$ & \\
\hline Other & $115(5.5 \%)$ & $112(7.1 \%)$ & $259(8.3 \%)$ & \\
\hline \multicolumn{5}{|l|}{ BMI $\left(\mathrm{kg} / \mathrm{m}^{2}\right)$} \\
\hline $18.5-24.9$ & $882(42.5 \%)$ & $657(42.5 \%)$ & $1201(38.8 \%)$ & .001 \\
\hline$<18.5$ & $91(4.4 \%)$ & $95(6.1 \%)$ & $163(5.3 \%)$ & \\
\hline $25.0-29.9$ & $479(23.1 \%)$ & $321(20.7 \%)$ & $665(21.5 \%)$ & \\
\hline 230.0 & $623(30.0 \%)$ & $665(21.5 \%)$ & $1063(34.3 \%)$ & \\
\hline Use of social services & $1297(61.6 \%)$ & $1025(65.4 \%)$ & $1910(61.1 \%)$ & .01 \\
\hline Tobacco use & $146(6.9 \%)$ & $122(7.8 \%)$ & $350(11.2 \%)$ & $<.001$ \\
\hline Prior preterm birth & $73(3.5 \%)$ & $104(6.6 \%)$ & $232(7.4 \%)$ & $<.001$ \\
\hline
\end{tabular}

Data are $N(\%)$.

BMI: body mass index; IPI: interpregnancy interval; PPROM: preterm premature rupture of membranes. 


\section{Table 2}

Multivariable analysis for the risk of developing PPROM based on IPI category.

\begin{tabular}{lcc}
\hline IPI Interval & ${\left.\text { Adjusted } \mathbf{O R}^{\boldsymbol{a}} \mathbf{( 9 5 \%} \mathbf{C I}\right)}^{\boldsymbol{p} \text { value }}$ \\
\hline IPI 6 months & $1.84(1.74-1.95)$ & $<.001$ \\
IPI 7-23 months & $1.05(0.99-1.12)$ & .10 \\
IPI 24 months & Ref & Ref \\
\hline
\end{tabular}

CI: confidence interval; IPI: interpregnancy interval; OR: odds ratio; PPROM: preterm premature rupture of membranes.

${ }^{a}$ Adjusted for maternal age, race, body mass index, use of social services, tobacco use, and history of preterm birth. 
Table 3

Association of each maternal characteristic with the risk of developing PPROM stratified by IPI.

\begin{tabular}{|c|c|c|c|c|}
\hline Characteristic & $\begin{array}{l}\text { IPI } \$ 6 \text { months }(N=93,674) \\
(\%)\end{array}$ & $\begin{array}{l}\text { IPI 7-23 months }(N= \\
125,761)(\%)\end{array}$ & $\begin{array}{l}\text { IPI } \geq 24 \text { months }(N=255,522) \\
(\%)\end{array}$ & OR (95\% CI) \\
\hline \multicolumn{5}{|l|}{ Maternal age (years) } \\
\hline$<35$ & $83,136(88.7)$ & $112,059(89.1)$ & $211,663(82.8)$ & Ref \\
\hline $35-44$ & $10,406(11.1)$ & $13,579(10.8)$ & $43,343(17.0)$ & $1.16(1.08-1.24)$ \\
\hline$\geq 45$ & $132(0.1)$ & $123(0.1)$ & $516(0.20)$ & $1.04(0.57-1.89)$ \\
\hline \multicolumn{5}{|l|}{ Maternal race } \\
\hline Caucasian & $70,158(74.9)$ & $98,397(78.2)$ & $192,617(75.4)$ & Ref \\
\hline African American & $15,600(16.6)$ & $16,512(13.1)$ & $36,998(14.5)$ & $2.18(2.06-2.31)$ \\
\hline Hispanic & $173(0.2)$ & $174(0.1)$ & $440(0.2)$ & $1.24(0.70-2.19)$ \\
\hline Asian & $938(1.0)$ & $1579(1.3)$ & $4081(1.6)$ & $0.92(0.73-1.16)$ \\
\hline Other & $6805(7.3)$ & $9099(7.2)$ & $21,386(8.4)$ & $1.08(0.99-1.19)$ \\
\hline \multicolumn{5}{|l|}{ BMI $\left(\mathrm{kg} / \mathrm{m}^{2}\right)$} \\
\hline $18.5-24.9$ & $38,954(42.1)$ & $56,221(45.2)$ & $106,550(42.2)$ & Ref \\
\hline$<18.5$ & $3602(3.9)$ & $5680(4.6)$ & $9029(3.6)$ & $1.41(1.26-1.57)$ \\
\hline $25.0-29.9$ & $21,683(23.4)$ & $27,919(22.4)$ & $58,915(23.3)$ & $0.99(0.93-1.05)$ \\
\hline 230.0 & $28,268(30.6)$ & $34,545(27.8)$ & $77,978(30.9)$ & $1.13(1.06-1.19)$ \\
\hline Use of social services & $56,452(60.3)$ & $65,883(52.4)$ & $131,6806(51.5)$ & $1.39(1.32-1.46)$ \\
\hline Tobacco use & $5428(5.8)$ & $7040(5.6)$ & $20,422(8.0)$ & $1.39(1.28-1.51)$ \\
\hline Prior preterm birth & $1539(1.6)$ & $2130(1.7)$ & $5091(2.0)$ & $3.59(3.24-3.98)$ \\
\hline
\end{tabular}

Data are $N(\%)$ of columns.

BMI: body mass index; CI: confidence interval; IPI: interpregnancy interval; OR: odds ratio; PPROM: preterm premature rupture of membranes. 


\section{Table 4}

Timing of delivery in gestational age categories by IPI among women with PPROM.

\begin{tabular}{|c|c|c|c|c|}
\hline $\begin{array}{l}\text { Gestational age } \\
\text { categories (weeks) }\end{array}$ & IPI 56 months $(N=\underset{(\%)}{2104)}$ & $\begin{array}{l}\text { IPI 7-23 months }(N=1566) \\
(\%)\end{array}$ & IPI $\geq 24$ months $(N=\underset{(\%)}{3127)}$ & $p$ value \\
\hline$\leq 24^{0}$ & $0(0.0)$ & $313(20.0)$ & 207 (6.6) & $<.001$ \\
\hline $24^{1}-28^{0}$ & $217(10.3)$ & $190(12.1)$ & $298(9.5)$ & .022 \\
\hline $28^{1}-32^{0}$ & $570(27.1)$ & $231(14.7)$ & $520(16.6)$ & $<.001$ \\
\hline $32^{1}-34^{0}$ & $632(30.0)$ & 355 (22.7) & $871(27.8)$ & $<.001$ \\
\hline $34^{1}-36^{6}$ & $685(32.6)$ & $477(30.5)$ & $1231(39.4)$ & $<.001$ \\
\hline
\end{tabular}

Data are $N(\%)$.

IPI: interpregnancy interval; PPROM: preterm premature rupture of membranes 